\title{
About Determining the Distribution of Air Bubbles in Water by Sizes Using a Passive Hydroacoustic Method
}

\author{
Stepan Gavrilev* and Mikhail Ivanov \\ B auman M oscow State Technical U niversity, Department of Ecology and Industrial Safety, M oscow, \\ Russia
}

\begin{abstract}
The task of determining the distribution of air bubbles in water by size is one of the keys in the calculation, design and operation of gasliquid systems. At present, optical and hydroacoustic methods are used to solve this problem. This paper describes the difficulties with the use of a passive hydroacoustic method. It is shown that the noise created by the bubbles is caused not only by their harmonic pulsations, but also by separation from the source and collapse. Upon separation and collapse, broadband noise is emitted, which complicates the allocation of the noise of the harmonic pulsations of the bubbles.
\end{abstract}

\section{Introduction}

Determining the distribution of air bubbles in water is one of the key tasks in the calculation, design and operation of gas-liquid systems. For example, when treating wastewater by flotation, both at the stage of preliminary calculation of the flotation stages and at the stage of maintaining the operating mode of the flotation unit, it becomes necessary to monitor the state of the gas-liquid mixture [1-3]. To solve such problems, optical and hydroacoustic systems are used.

Optical methods for determining the size of gas bubbles in liquid media are based on the principles of light blocking [4] or reflection in a dark field [5]. The essence of these methods is that a laser beam is passed through the bubble and the reflected and refracted light from the bubble is measured by a photodetector. Optical methods also include photometric methods. They are based on the analysis of images of a gas-liquid mixture, for example, using the DensePose method of computer vision [6]. The correctness of the bubble's definition depends on many factors. First of all, on the quality of images (resolution and contrast), it should be sufficient so that you can distinguish a single bubble. Secondly, from the applied snapshot analysis algorithm.

The main drawback of optical and photometric methods is that they are applicable only in transparent optical media, i.e. they are not suitable for measurements in turbid liquid media. The wastewater is often turbid, therefore acoustic methods are used in such cases.

* Corresponding author: stepan.tab92@ gmail.com 
Most modern acoustic methods are active, i.e. they are based on measuring the attenuation and velocity of an acoustic wave passing through a bubble cloud layer. Examples of the implementation of this approach are described in [7-9]. But it is worth considering that such methods have an important feature: an acoustic wave generated to irradiate a bubble cloud can affect the bubbles. This phenomenon can be seen in the results of [13]. The authors of this work conducted a series of experiments, while changing the intensity of the emitted acoustic wave and in each of the experiments received different results. Thus, in a number of cases where maintaining modes with a certain bubble size is especially important, the use of active methods is unacceptable. Therefore, it is preferable to use passive methods that do not affect the dynamics of the studied processes.

\section{Theoretical part}

The issue of bubble acoustics was first considered by Lord Rayleigh in 1917 in [10]. In this work, he considered the dynamics of a single gas bubble in an incompressible fluid. The bubble pulsates under the influence of a sound wave. Rayleigh described the dynamics of the bubble by the following equation:

$$
\rho_{1}\left(R \ddot{R}+\frac{3}{2} R^{2}\right)=P_{R}(t)-P_{\infty}(t),
$$

where $R$ is the radius of the bubble, $\rho_{1}$ is the density of the liquid, $P_{R}(t)$ is the time-varying pressure on the surface of the bubble, $P_{\infty}(t)$ is the pressure at infinity.

In 1933, Minnaert showed that under adiabatic conditions (heat transfer between the gas of the bubble and the liquid is insignificant), the frequency of the acoustic signal $f$ emitted by the gas bubble depends on its size as follows:

$$
f=\frac{1}{2 \pi R} \sqrt{\frac{3 \gamma P}{\rho}},
$$

where $\mathrm{f}$ is the frequency of the sound wave emitted by the bubble; $\mathrm{P}$ is the absolute pressure of the liquid; $\gamma$ is the coefficient of gas specific heat, $\rho$ is the density of the liquid; $R$ is the bubble radius.

This expression is valid for spherical bubbles. The shape of the bubbles usually changes during the ascent. Depending on the size, they can take the form of a sphere, a flattened spheroid of a spherical segment or a mushroom cap. In [11], a criterion was presented in accordance with which bubbles can be considered approximately spherical if the condition:

$$
R<\delta_{\sigma}
$$

where $R$ is the bubble radius; $\delta$ is the capillary constant of the liquid.

According to this criterion, the bubbles in the water have the shape of a sphere if their radius $R<2.7 \mathrm{~mm}$.

Minnaert's expression also shows a very simple inverse relationship for calculating the size of a bubble from the frequency of the sound it emits. For example, if an air bubble in water emits sound at a frequency of $3000 \mathrm{~Hz}$, then its radius is $1 \mathrm{~mm}$.

The most complete description of bubble acoustics was given by Leighton (1994). According to [12], the spectrum of sound emitted by bubbles can be used to determine their size. Then this idea was developed in [13-19]. But all the works do not take into account the fact that the bubbles create noise not only as a result of pulsations, but also when they are separated from the source and collapse. 


\section{Experimental part}

To clearly reflect the need to separate the total noise of air bubbles in water, a number of experiments were carried out. The installation consists of: a transparent column (1) with a height of $1200 \mathrm{~mm}$ and a diameter of $50 \mathrm{~mm}$, an aerator (2), a cylinder of compressed air (3), two hydrophones of 8103 type from Bruel \& Kjaer (5), a multi-channel signal analyzer Pulse LAN-XI from Bruel \& Kjaer (6) and PC (7) with LabShop software.

Column (1) was filled with water. Air was supplied into it from a cylinder (3) through an aerator (2). Air flow was set by the valve (4). Bubble noise was measured by hydrophones (5) connected to a multichannel spectrum analyzer (6). Measurements were processed on a PC (7) with Labshop software. One of the hydrophones was located at the bottom of the column in close proximity to the aerator. The second hydrophone moved freely along the entire height of the column.

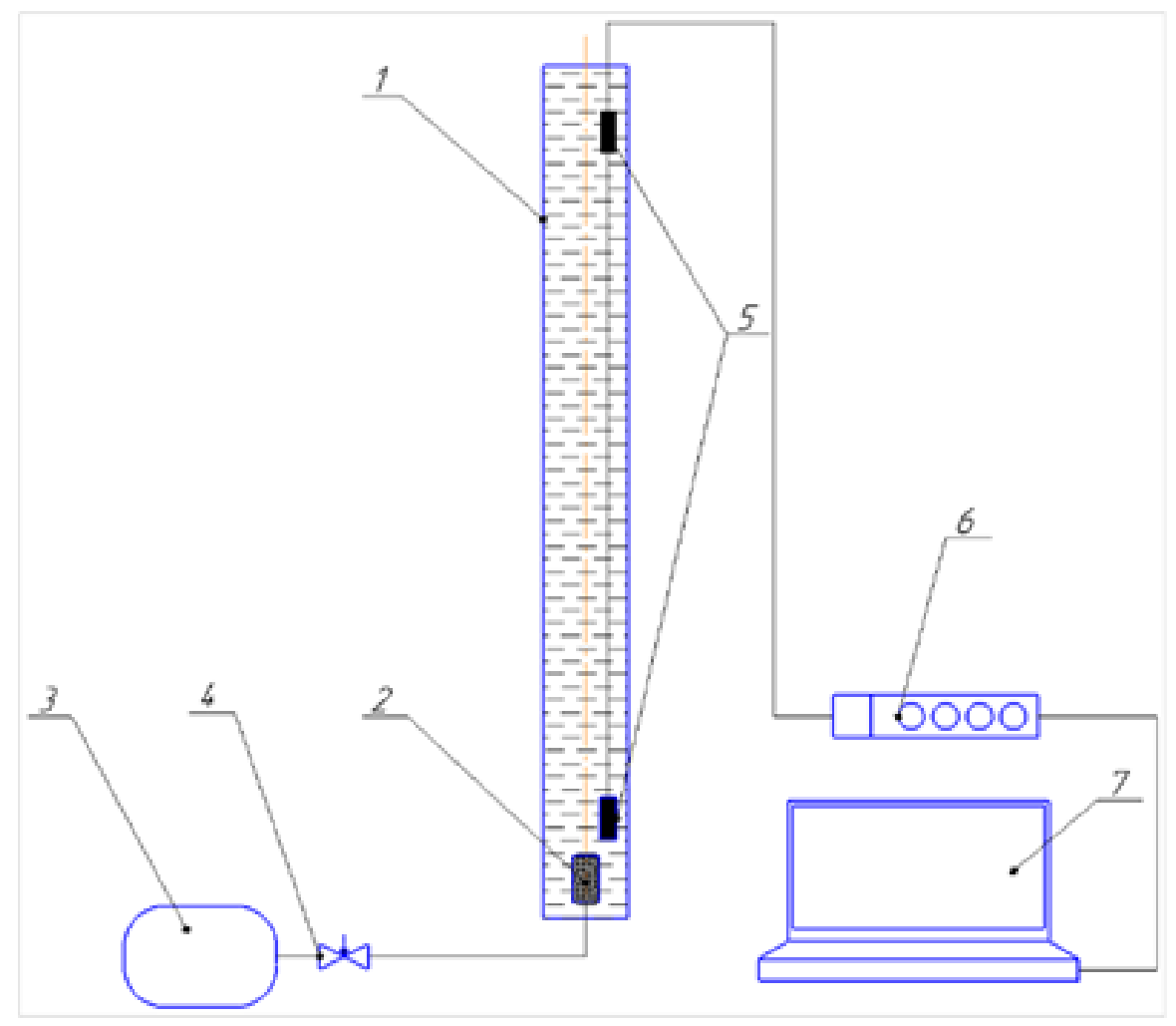

Fig 1. Experimental stand layout

The mean bubble radius was $\sim 2 \mathrm{~mm}$. It was evaluated photometrically. Since the bubbles were approximately the same size, based on expression 2, we expected to see peaks on the spectra at a frequency of $\sim 1.5 \mathrm{kHz}$. But if you look at the measurement results (Fig. 2), then the peak at this frequency can be detected only on the spectrum obtained from measurements in the middle of the column.

At the bottom of the column, the noise level created by the bubbles is higher than in the middle and upper point of the column and has a broadband spectrum (up to $\sim 4.7 \mathrm{kHz}$ ). In the middle of the column, the high-frequency part of the separation noise attenuates, and it is already possible to distinguish a peak at a frequency of $\sim 1.5 \mathrm{kHz}$, corresponding to the pulsations of the bubbles. And at the highest point of the column, the spectrum again acquires 
a broadband character (up to $\sim 4 \mathrm{kHz}$ ), which does not allow us to select the peak we need. Presumably, this is the noise of the collapse of the bubbles.

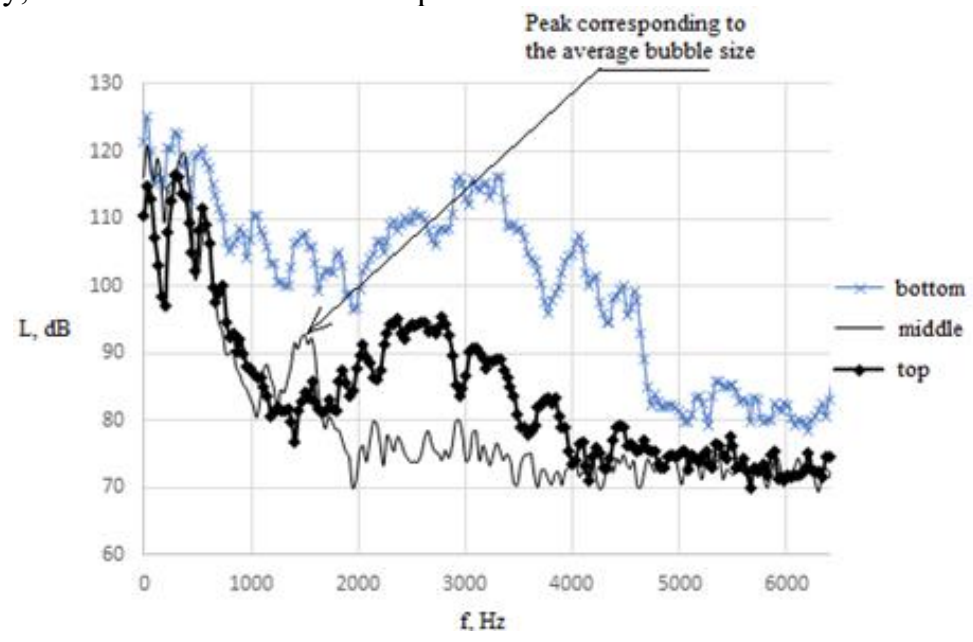

Fig 2. Experiment results

\section{Conclusion}

In this paper, it is clearly shown that the noise created by the bubbles is caused not only by their harmonic pulsations, but also by separation from the source and collapse. Upon separation and collapse, broadband noise is emitted, which complicates the allocation of the noise of the harmonic pulsations of the bubbles. In some cases, this task can be simplified by isolating with distance, i.e. it is necessary to select the installation point of the measuring hydrophones, or use a vertical array of hydrophones (taking into account the application experience described in [20]) so that the separation and collapse noise levels are sufficiently lower than the noise level of bubble pulsations. And also knowing in advance the maximum possible size of the bubbles and the fact that the pulsation frequency of the bubbles, according to Minnaert, is inversely proportional to their size, it is possible to get rid of the lowfrequency part of the spectrum by applying low-pass filters.

\section{References}

1. B. S. Ksenofontov and M. V. Ivanov, Water Sci. Technol. (2013).

2. M. V Ivanov and B. S. Ksenofontov, Water Sci. Technol. 69, 1434 (2014).

3. D. Sazonov, B. Ksenofontov, E. Antonova, and V. Yushinov, in Int. Multidiscip. Sci. GeoConference Surv. Geol. Min. Ecol. Manag. SGEM (International Multidisciplinary Scientific Geoconference, 2014), pp. 815-820.

4. Y. Zhang and H. Sun, Proc. - Int. Conf. Commun. Syst. Netw. Technol. CSNT 2012 671 (2012).

5. Martin Villiger, Christophe Pache, Noelia Bocchio, Joan Goulley, and Theo Lasser, in Biomed. Opt. (2010).

6. A. Zhuravlev, in IEEE Int. Conf. Microwaves, Antennas, Commun. Electron. Syst. (2019), pp. 1-6.

7. X. J. Wu and G. L. Chahine, J. Hydrodyn. 22, 325 (2010).

8. M. Christensen and P. Thomassen, 121 (2014).

9. J. Xue, 1 (2004). 
10. Lord Rayleigh, London, Edinburgh, Dublin Philos. Mag. J. Sci. (1917).

11. P. Volkov, Gas-liquid mechanics 75 (1996).

12. T. Leighton, (1994).

13. J. W.R. Boyd and J. Varley, Chem. Eng. Sci. 56, 1749 (2001).

14. R. Manasseh, R. F. LaFontaine, J. Davy, I. Shepherd, and Y.-G. Zhu, Exp. Fluids 30, 672 (2001).

15. C. A. Greene and P. S. Wilson, J. Acoust. Soc. Am. 131, EL61 (2012).

16. S. Husin and D. Mba, II, 0 (2010).

17. M. Devaud, T. Hocquet, J. Bacri, V. Leroy, M. Devaud, T. Hocquet, J. Bacri, and V. Leroy, (2007).

18. W. Kracht and C. Moraga, Miner. Eng. 98, 122 (2016).

19. S. A. Gavrilev, M. V Ivanov, and E. A. Yusupov, in Lect. Notes Eng. Comput. Sci. Proc. World Congr. Eng. 2019, 3-5 July, 2019, London, U.K. (2019), pp. 527-530.

20. M. V. Volkov, V. A. Grigoriev, A. A. Lunkov, and V. G. Petnikov, Acoust. Phys. 65, 269 (2019). 